\title{
Effect of cataract extraction on frequency doubling technology perimetry in patients with glaucoma
}

\author{
M A R Siddiqui, A Azuara-Blanco, S Neville
}

Br J Ophthalmol 2005;89:1569-1571. doi: 10.1136/bjo.2005.080655

Aim: To evaluate the effect of cataract surgery on frequency doubling technology (FDT) perimetry in patients with coexisting cataract and glaucoma.

Methods: In this consecutive prospective cohort study 27 patients with open angle glaucoma scheduled for cataract extraction alone or combined with trabeculectomy were enrolled. All patients underwent FDT threshold C-20 visual fields within 3 months before and 3 months after surgery. Changes in mean deviation (MD) and pattern standard deviation (PSD) were evaluated. Additionally, changes in best corrected logMAR visual acuity (VA), intraocular pressure (IOP), and number of glaucoma medications were also studied.

Results: 22 patients completed the study. VA improved after surgery, from 0.47 (SD 0.19) to $0.12(0.17)(p<0.001)$. The visual indexes changed after cataract extraction: $M D$ improved (from -10.9 (SD 4.6) dB to -7.0 (4.6) dB; $\mathrm{p}<0.001$ ) while PSD worsened (from 7.1 (SD 3.5) $\mathrm{dB}$ to 8.5 (3.8) $d B ; p=0.001$ ).

Conclusion: In patients with co-existing cataract and glaucoma, examined with FDT, MD improved and PSD worsened after cataract surgery. Global indexes of FDT should be interpreted with caution in patients with glaucoma and cataracts.

$\mathrm{V}$ isual field (VF) assessment provides essential information for the diagnosis and management of glaucoma. In the past decade novel VF tests such as frequency doubling technology (FDT), have emerged. FDT utilises the frequency doubling illusion, in which a sine wave grating of low spatial frequency ( $<1$ cycle/degree) undergoing counterphase flicker at high temporal frequency $(>15 \mathrm{~Hz})$ appears to the observer to have double the number of bars than are actually present. ${ }^{1}$ This psychophysical illusion is thought to be mediated by $\mathrm{M}_{\mathrm{y}}$ ganglion cells which are a subset of the magnocellular system. ${ }^{2}$

FDT has been released commercially and provides suprathreshold and threshold strategies. Suprathreshold modes (C-20-5 and C-20-1), requiring less than 1 minute per eye, have shown good diagnostic performance and have been used for glaucoma screening. ${ }^{3}{ }^{4}$ Another positive aspect of FDT is that it may be able to detect glaucomatous field loss earlier than the standard white on white perimetry, ${ }^{56}$ perhaps because large diameter magnocellular ganglion cells may be preferentially damaged early in glaucoma. ${ }^{7}$

Glaucoma and cataract have an increased prevalence in elderly populations and they often co-exist. Evaluating the results of VF tests in patients with cataract and glaucoma is a common challenge. Previous studies have shown the effect of cataract on standard white on white perimetry in patients with glaucomatous VF loss. ${ }^{8-13}$ Similarly, cataract has been shown to affect short wavelength automated perimetry (SWAP). ${ }^{14}$ Recent work has demonstrated the effect of cataract on FDT perimetry in normal subjects. ${ }^{15}{ }^{16}$ The aim of this study was to evaluate the effect of cataract extraction on FDT perimetry results in patients with glaucoma.

\section{PATIENTS AND METHODS}

Glaucoma patients were prospectively recruited from the glaucoma unit in an academic hospital. Patients included in this study were scheduled for cataract extraction alone or in combination with trabeculectomy. The indication for cataract surgery was visually significant cataract that was thought to contribute to limitations in daily activities. The indication for phaco-trabeculectomy included uncontrolled intraocular pressure or medically controlled intraocular pressure with more than two medications and advanced glaucoma related damage to the optic nerve associated with a visually significant cataract. The study adhered to the tenets of the Declaration of Helsinki and informed consent was obtained from all participants. Ethics committee approval was available for this study.

Patients with chronic open angle glaucoma (primary open angle glaucoma, normal tension glaucoma, pseudoexfoliation glaucoma, or pigmentary glaucoma) with a Snellen visual acuity (VA) of $6 / 36$ or better were included in the study. The diagnosis of glaucoma was based on the presence of glaucomatous optic disc cupping (for example, cup to disc ratio of more than 0.8 , disc asymmetry, thinning of the neuroretinal rim). Exclusion criteria included the use of miotics drops, ocular morbidity other than glaucoma or cataract, and inability to perform reliable white on white VF (more than 33\% of false positive, false negatives, or fixation losses). Patients with surgical complications, consistent postoperative IOP of more than $35 \mathrm{~mm} \mathrm{Hg}$, or unreliable FDT tests (more than 33\% abnormal responses on any of the reliability indexes) were also excluded.

Threshold FDT C-20 perimetry (Carl-Zeiss Meditec, Dublin, CA, USA; and Welch Allyn, Skaneateles Falls, NY, USA) was performed within 3 months before and after surgery. A screening FDT test was used in each patient as a training tool. All eyes were refracted 1 month after surgery. Best corrected logMAR acuity, using EDTRS vision charts under appropriate illumination, and IOP, using Goldmann applanation tonometry, were evaluated at the time of preoperative and postoperative VF testing. The number of antiglaucoma medications was also recorded.

All patients undergoing phacoemulsification had a clear cornea procedure with a foldable intraocular lens implant (MA60BM, Alcon Surgical, Fort Worth, TX, USA) under topical anaesthesia. Patients undergoing combined

Abbreviations: FDT, frequency doubling technology; IOP, intraocular pressure; MD, mean deviation; PSD, pattern standard deviation; SWAP, short wavelength automated perimetry; VA, visual acuity; VF, visual fields 


\begin{tabular}{ll} 
Table 1 & Participant demographics \\
\hline Age, mean (SD) (range) years & $76.6(8.4)(54-90)$ \\
Sex (male: female ratio) & $13: 9$ \\
Race (white: non-white ratio) & $22: 0$ \\
Type of glaucoma & \\
POAG, n (\%) & $19(86.4 \%)$ \\
PXF, $n(\%)$ & $2(9.1 \%)$ \\
NTG, $n(\%)$ & $1(4.5 \%)$ \\
Type of surgery & $16(72.7 \%)$ \\
Phaco-trabeculectomy, $\mathrm{n}(\%)$ & $6(27.3 \%)$ \\
Phacoemulsification, $\mathrm{n}(\%)$ &
\end{tabular}

POAG, primary open angle glaucoma; PXF, pseudoexfoliation glaucoma; NTG, normal tension glaucoma.

phaco-trabeculectomy had a one site procedure under subTenon's anaesthesia. A fornix based conjunctival dissection was made and $0.4 \mathrm{mg} / \mathrm{ml}$ of mitomycin C (MMC) was applied with soaked sponges over the bare sclera and covered with the conjunctival flap for at least 1 minute. The area exposed to MMC was vigorously irrigated with balanced salt solution. A $4 \mathrm{~mm} \times 3 \mathrm{~mm}$ partial thickness rectangular scleral flap was constructed in the superior limbus. A block of corneal tissue was removed with a punch and a small peripheral iridectomy was performed. Phacoemulsification was carried out before removing the block of tissue. The scleral flap was sutured with at least two interrupted and one releasable 10/0 nylon sutures. The conjunctival flap was sutured watertight with 10/0 nylon sutures. Ocular massage, laser suturelysis, and 5-fluorouracil subconjunctival injection was carried out according to the surgeon's criteria.

Data were entered in a statistical software package SPSS (version 10-0, Chicago, IL, USA). Normality of the data was assessed by Kolmogorov-Smirnov test. Changes between preoperative and postoperative VF indexes were evaluated by paired $t$ test. Associations between improvement of VA and changes in MD and PSD values of FDT after cataract extraction were analysed with the Pearson correlation test. Probability values of less than 0.05 were considered to be statistically significant.

\section{RESULTS}

Twenty seven patients were enrolled in the study. Two patients failed to attend for postoperative VF test within 3 months. One patient complained of a red and watery eye after surgery and was unable to perform the test postoperatively. Another patient died of unrelated ruptured abdominal aortic aneurysm 2 months after surgery. One patient developed visual distortion after surgery and was found to have a choroidal neovascular membrane. A total of 22 patients were included in the final analysis.

Demographics of the participants are summarised in table 1. Changes in VA, IOP, and number of glaucoma medications are shown in table 2. The median number of antiglaucoma medications was two before surgery and none

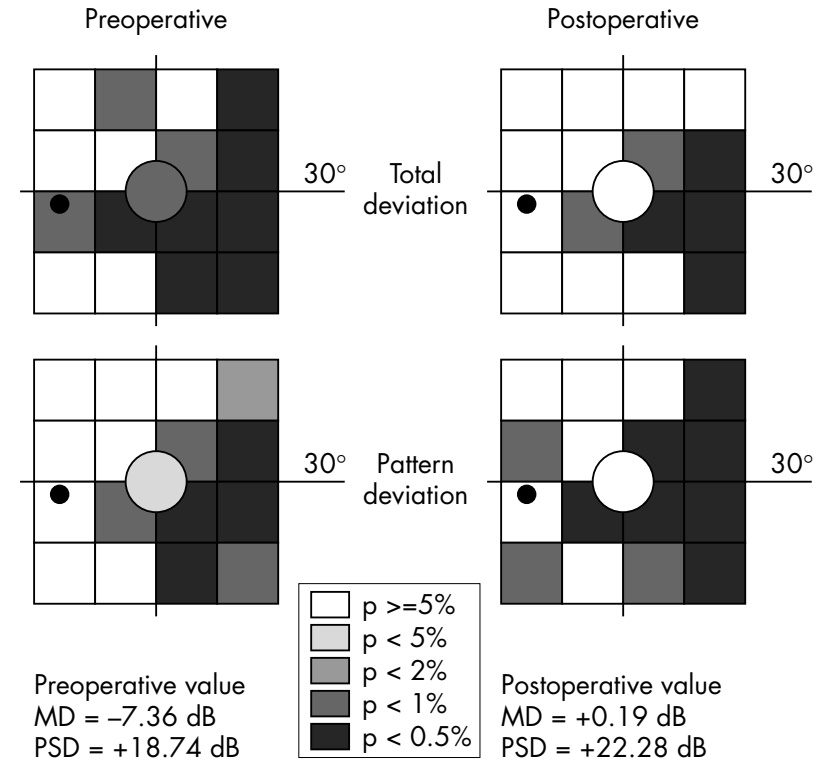

Figure 1 FDT perimetry printout (total and pattern deviation plots, and $M D$ and PSD values) before and after surgery of a patient with glaucoma and cataract who underwent phaco-trabeculectomy.

after surgery. In patients undergoing phaco-trabeculectomy $(\mathrm{n}=16)$ the mean IOP (SD) before and after surgery was $20.2(5.0)$ and $15.9(3.7)$, respectively $(\mathrm{p}=0.014)$.

The MD value improved after surgery $(p<0.001)$, while PSD deteriorated $(p=0.001)$ (fig 1$)$. These changes were statistically significant (table 2 ). The extent of VA improvement correlated with the deterioration of PSD score. The Pearson correlation test showed a statistically significant correlation between the postoperative VA improvement and the PSD change $\left(\mathrm{p}=0.024, R^{2}=0.478\right)$. However, the changes of MD and VA were not correlated $(p=0.252$, $R^{2}=0.252$ ).

\section{DISCUSSION}

In white on white conventional perimetry cataract usually results in a diffuse reduction of sensitivity with a worsening of the MD index. However, indexes designed to quantify localised defects would not expected to be affected by cataract. Several studies have evaluated the effects of cataract on white on white perimetry in normal subjects and also in patients with glaucoma. In the majority of evaluations on normal volunteers an improvement in MD was noted after cataract surgery, while the PSD remained unchanged. ${ }^{16-18}$ However, in glaucoma patients examined with standard perimetry a worsening of PSD was observed after cataract extraction (table 3$).^{9-13}$

Table 2 Changes in VA, IOP, number of glaucoma medications, and FDT global indexes

\begin{tabular}{|c|c|c|c|}
\hline & \multirow{2}{*}{$\frac{\text { Preoperative }}{\text { Mean (SD) }}$} & \multirow{2}{*}{$\begin{array}{l}\text { Postoperative } \\
\text { Mean (SD) }\end{array}$} & \multirow{2}{*}{$\begin{array}{l}\text { p Value(paired } \\
t \text { test) }\end{array}$} \\
\hline & & & \\
\hline $\begin{array}{l}\text { Best corrected logMAR acuity } \\
\text { IOP (all patients, } n=22)(\mathrm{mm} \mathrm{Hg} \text { ) } \\
\text { IOP (phaco-trabeculectomy, } \mathrm{n}=16)(\mathrm{mm} \mathrm{Hg} \text { ) } \\
\text { No of medications (all patients, } \mathrm{n}=22 \text { ) } \\
\text { No of medications (phaco-trabeculectomy, } \mathrm{n}=16 \text { ) } \\
\text { Mean deviation ( } \mathrm{dB} \text { ) } \\
\text { Pattern standard deviation (dB) }\end{array}$ & $\begin{array}{l}0.47(0.19) \\
18.8(5.5) \\
20.2(5.0) \\
1.8(0.8) \\
2.2(0.5) \\
-10.9(4.6) \\
7.1(3.5)\end{array}$ & $\begin{array}{l}0.12(0.17) \\
16.6(4.0) \\
15.9(3.7) \\
0.5(0.7) \\
0.2(0.5) \\
-7.0(4.6) \\
8.5(3.8)\end{array}$ & $\begin{array}{l}<0.001 \\
0.063 \\
0.014 \\
<0.001 \\
<0.001 \\
<0.001 \\
0.001\end{array}$ \\
\hline
\end{tabular}


Table 3 Studies assessing effect of cataract on visual field tests

\begin{tabular}{|c|c|c|c|c|c|}
\hline Study & Year & Test & Glaucoma & $M D^{*}$ & CPSD, PSD, or CLV* \\
\hline Lam et $a l^{17}$ & 1991 & Humphrey threshold 30-2 & No & $\uparrow$ & $\leftrightarrow$ \\
\hline Yao et al ${ }^{18}$ & 1993 & Octopus (program G1) & No & $\uparrow$ & $\leftrightarrow(\mathrm{CLV})$ \\
\hline Stewart et $a^{\beta}$ & 1995 & Humphrey $30-2$ & Yes & $\leftrightarrow$ & $\leftrightarrow$ \\
\hline Smith et al & 1997 & Humphrey threshold $24-2$ or $30-2$ & Yes & $\uparrow$ & $\downarrow$ \\
\hline Chen et $a l^{10}$ & 1998 & Humphrey threshold $24-2$ or $30-2$ & Yes & $\uparrow$ & $\downarrow$ \\
\hline Gillies et $a l^{11}$ & 1998 & Humphrey threshold 24-2 & Yes & $\uparrow$ & $\downarrow$ \\
\hline Kim et $a l^{14}$ & 2001 & White-on-white 24-2 (FASTPAC) & No & $\uparrow$ & $\leftrightarrow$ \\
\hline Kim et $a l^{14}$ & 2001 & Blue on yellow 24-2 (FASTPAC) & No & $\uparrow$ & $\leftrightarrow$ \\
\hline Hayashi et $a l^{22}$ & 2001 & Humphrey threshold 30-2 & Yes & $\uparrow$ & $\downarrow$ \\
\hline Kook et $a l^{16}$ & 2004 & FDT C-20 & No & $\uparrow$ & $\leftrightarrow$ \\
\hline Kook et al ${ }^{16}$ & 2004 & SITA-fast 30-2 & No & $\uparrow$ & $\leftrightarrow$ \\
\hline Tanna et $a l^{15}$ & 2004 & FDT threshold C-20 & No & $\uparrow$ & $\leftrightarrow$ \\
\hline Koucheki et $a l^{3}$ & 2004 & Humphrey threshold 24-2 & Yes & $\uparrow$ & $\downarrow$ \\
\hline Our study & 2005 & FDT threshold C-20 & Yes & $\uparrow$ & $\downarrow$ \\
\hline
\end{tabular}

*Post-cataract extraction: $(\leftrightarrow)$ no change; $(\uparrow)$ improved; $(\downarrow)$ worsened.

MD, mean deviation; PSD, pattern standard deviation; CPSD, corrected pattern standard deviation; CLV, corrected loss variance.

The impact of cataract on the results of FDT perimetry has been evaluated recently in normal subjects. Tanna et al ${ }^{15}$ found that cataract has an adverse effect on MD but not on PSD. Similarly, Kook et al ${ }^{16}$ evaluated the effect of cataract in healthy individuals undergoing both FDT threshold C-20 perimetry and SITA-fast. Their results showed a generalised reduction of sensitivity in FDT and SITA-fast associated with cataract, whereas PSD remained unchanged in both tests. However, to our knowledge, there are no reports on the effect of cataract on FDT in patients with glaucoma. As it has been shown that FDT perimetry is resistant to refractive blur, ${ }^{19}$ researchers have thought that it may also be resistant to the effects of cataract to some extent. It has been suggested that, in screening mode of FDT, cataracts are not a cause of abnormal results. ${ }^{20}$

In the current study, among patients with cataract and glaucoma examined with FDT perimetry both MD and PSD indexes changed after cataract surgery. The improvement of MD was not surprising. However, the PSD worsening after cataract extraction highlights the potential of underestimating the true extent of VF defects on sequential observations in glaucoma patients. This finding has also been observed in standard white on white full threshold perimetry (see above). It may be possible that a cataract renders margins of a localised field defect less distinct, hence masking "true" PSD. It is difficult to attribute these changes to a learning effect. Previous studies evaluating the learning effect of patients with glaucoma using FDT did not show any differences in PSD values. ${ }^{21}$

FDT has been advocated as a useful test for screening in glaucoma. Because PSD may be masked by cataract, some patients with increased MD but "normal" PSD may have glaucoma. This could diminish the sensitivity of FDT to detect glaucoma in patients with co-existing cataract. As cataract also worsens MD, it could affect the specificity of the test in patients with only cataract.

A limitation of our study was that a standard system to quantify lenticular opacities was not used. The effect of lens opacities on VF tests may vary with the type of cataract. Similarly, the sample size was small and it was not possible to analyse the effects of different severities and types of glaucoma on FDT parameters.

In conclusion, FDT is affected by lens opacities in glaucoma patients. Global indexes of FDT should be used with caution in patients with coexisting cataract and glaucoma.

\section{Authors' affiliations}

M A R Siddiqui, A Azuara-Blanco, S Neville, Department of Ophthalmology, NHS Grampian, University of Aberdeen AB25 2ZN, UK

\section{Competing interests: none declared}

Correspondence to: Augusto Azuara-Blanco, PhD, FRCS(Ed), The Eye Clinic, Aberdeen Royal Infirmary, Aberdeen AB25 2ZN, UK. aazblanco@aol.com

Accepted for publication 21 August 2005

\section{REFERENCES}

1 Kelly DH. Frequency doubling in visual responses. J Opt Soc Am A 1966:56:1628-33.

2 Johnson CA, Samuels SJ. Screening for glaucomatous visual field loss with frequency-doubling perimetry. Invest Ophthalmol Vis Sci 1997;38:413-25.

3 Quigley HA. Identification of glaucoma-related visual field abnormality with the screening protocol of frequency doubling technology. Am J Ophthalmol 1998; 125:819-29.

4 Wadood AC, Azuara-Blanco A, Aspinall P, et al. Sensitivity and specificity of frequency-doubling technology, tendency-oriented perimetry, and Humphrey Swedish interactive threshold algorithm-fast perimetry in a glaucoma practice. Am J Ophthalmol 2002;133:327-32.

5 Sample PA, Bosworth CF, Blumenthal EZ, et al. Visual function-specific perimetry for indirect comparison of different ganglion cell populations in glaucoma. Invest Ophthalmol Vis Sci 2000;41:1783-90.

6 Medeiros FA, Sample PA, Weinreb RN. Frequency doubling technology perimetry abnormalities as predictors of glaucomatous visual field loss. Am J Ophthalmol 2004;137:863-71.

7 Quigley HA, Dunkelberger GR, Green WR. Chronic human glaucoma causing selectively greater loss of large optic nerve fibers. Ophthalmology 1988:95:357-63.

8 Stewart WC, Rogers GM, Crinkley CM, et al. Effect of cataract extraction on automated fields in chronic open-angle glaucoma. Arch Ophthalmol 1995; 113:875-9.

9 Smith SD, Katz J, Quigley HA. Effect of cataract extraction on the results of automated perimetry in glaucoma. Arch Ophthalmol 1997;115:1515-19.

10 Chen PP, Budenz DL. The effects of cataract extraction on the visual field of eyes with chronic open-angle glaucoma. Am J Ophthalmol 1998; 125:325-33.

11 Gillies WE, Brooks AM. Effect of lens opacity on the glaucomatous field of vision. Aust N Z J Ophthalmol 1998;26(suppl):19-21.

12 Hayashi K, Hayashi H, Nakao F, et al. Influence of cataract surgery on automated perimetry in patients with glaucoma. Am J Ophthalmol 2001;132:41-6.

13 Koucheki B, Nouri-Mahdavi K, Patel G, et al. Visual field changes after cataract extraction: the AGIS experience. Am J Ophthalmol 2004;138:1022-8.

$14 \mathrm{Kim}$ YY, Kim JS, Shin DH, et al. Effect of cataract extraction on blue-on-yellow visual field. Am J Ophthalmol 2001;132:217-20.

15 Tanna AP, Abraham C, Lai J, et al. Impact of cataract on the results of frequency-doubling technology perimetry. Ophthalmology 2004; 111:1504-7.

16 Kook MS, Yang SJ, Kim S, et al. Effect of cataract extraction on frequency doubling technology perimetry. Am J Ophthalmol 2004;138:85-90.

17 Lam BL, Alward WL, Kolder HE. Effect of cataract on automated perimetry. Ophthalmology 1991;98:1066-70.

18 Yao K, Flammer J. Relationship cataract density and visual field damage. Eur J Ophthalmol 1993;3:1-5.

19 Anderson AJ, Johnson CA. Frequency-doubling technology perimetry and optical defocus. Invest Ophthalmol Vis Sci 2003;44:4147-52.

20 Sponsel WE, Trigo Y, Hendricks J, et al. Frequency doubling perimetry. Am J Ophthalmol 1998;126:155-6.

21 Matsuo H, Tomita G, Suzuki Y, et al. Learning effect and measurement variability in frequency-doubling technology perimetry in chronic open-angle glaucoma. J Glaucoma 2002;11:467-73. 